\title{
A STUDY OF LEVEL OF COMMON CAROTID ARTERY BIFURCATION IN RELATION TO THE VERTEBRAL BODY LEVEL IN A COHORT OF PATIENTS REFERRED TO THE ANGIOGRAPHY SECTION OF NEURO TRAUMA CENTRE, NATIONAL HOSPITAL OF SRI LANKA.
}

\author{
Athukorala A D P ${ }^{1}$,Maniharan $\mathrm{S}^{1}$, Guruge S I T ${ }^{1}$, Lekamge I N ${ }^{1}$ \\ Department of Radiology, National Hospital of Sri Lanka ${ }^{1}$
}

Keywords :Carotid bifurcation, Internal carotid artery, vertebral body level, Digital subtraction angiography

Corresponding Author:Athukorala A D $P^{1}$

Copyright:Athukorala A $D P^{1}$

https://orcid.org/0000-0002-8502-9085

\begin{abstract}
\section{Background and Objective}

The carotid bifurcation (CB) is an important anatomical landmark and it is essential to have a thorough knowledge regarding the levels at which it bifurcates occurs in relation to the vertebral body level, especially in the fields of interventional radiology and surgery.
\end{abstract}

\section{Methodology}

Lateral projection of common carotid run with bony overlay was used to evaluate the level of $\mathrm{CB}$ in relation to 13 pre-determined levels. Images of 287 patients; 129 males and 158 females, from 6 to 88 years, who underwent digital subtraction cerebral angiography at a single center in Sri Lanka were included in study.

\section{Results}

Highest CB level was observed at right C2 in both males $(n=1,0.4 \%)$ and females $(n=15$, 4.9\%). The lowest in males was seen at left $C 6 / 7(n=1,0.4 \%)$ and in females at $C 5 / 6(n=1$, $0.3 \%)$. C3 was the commonest CB level $(n=174,31.4 \%)$. CB level was found to be significantly lower in males $(U=30109.5, p<0.001)$ and lower on left side $(U=33850.5, p=$ 0.014). Bilaterally symmetric CBs were seen in 136 (50.9\%), which was commoner among females (M:F of 1:1.3) and occurred most frequently at C3 $(n=49,18.4 \%)$.

Average mid-cervical internal carotid artery (ICA) diameter was $4.1 \mathrm{~mm} \pm 0.9 \mathrm{~mm}$ and $4.0 \mathrm{~mm}$ $\pm 0.8 \mathrm{~mm}$ and length was between $3.5 \mathrm{~cm}-12.0 \mathrm{~cm}$ and $2.9 \mathrm{~mm}$ to $-10.9 \mathrm{~mm}$ in males and females respectively. No correlation was found between neck length and the level of CB ( $\mathrm{p}=$ 0.099).

\section{Conclusion}

The CB level ranging from the highest at C2 to the lowest at C6/7 with the most frequent level of bifurcation being at C3 corresponds to the other available international studies. Similarly, the prevalence of symmetric CBs was also comparable. Mean ICA diameter in our study was lower compared to other studies and may prove to be a useful predictor in local clinical practice.

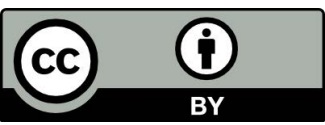




\section{Background and introduction}

The carotid bifurcation (CB) is an important anatomical landmark that is relevant to both physiological and pathological processes. It has been recognized that $\mathrm{CB}$ exhibits a great degree of variability in its position in the neck as a consequence of its embryologic origins. Given that interventions are carried out in and around the region of the CB in diverse fields such as vascular surgery, neurosurgery and ENT surgery as well as in interventional radiology it is essential to have a thorough knowledge regarding the range of levels at which carotid bifurcation occurs.Being one of the commonest sites for atherosclerotic narrowing (1), the carotid bifurcation remains a frequent target for both vascular surgical and endovascular interventions. With the rising trend of metabolic syndrome in the wider Asian region (2) the incidence of carotid atherosclerosis needing intervention is likely to rise in future. In addition it is recognized that both vascular surgical and endovascular interventions for atherosclerotic narrowing have a relatively high morbidity and mortality (3) with ischaemic stroke and myocardial infarctions being the most serious complications, which are associated with endovascular intervention and vascular surgery respectively.(4)

From an endovascular interventionist's perspective, a primary importance of understanding the variations in CB level lies in planning for carotid stenting. (5) Given the fact that significant variations exist in the sizes of organs and vessels among various populations, knowledge on ICA dimensions in local patients such as its diameter and length will be of importance when deciding on the stent caliber and length.

From a vascular surgical perspective, identifying the carotid bifurcation is of paramount importance when carrying out interventions such as endarterectomies, which enable the operating surgeon to trace the CCA and ICA downwards and upwards respectively. Proper identification of the CB is important in order to differentiate CCA from ICA as neither of these vessels have side branches. Surgical intervention following accidental incorrect identification may result in disastrous consequences for the patient and hence all possible steps should be taken to avoid such an outcome.

In consideration of the above, the aim of this study was to evaluate the carotid bifurcation level and the diameter and length of cervical ICA in a cohort of patients presented for cerebral angiographies at the Neurotrauma Centre, National Hospital of Sri Lanka. The information obtained on the local population from this study will be of value to specialists in multiple fields, including surgery and endovascular interventionists in their daily practice as mentioned above. It may also would be a useful base upon which future local studies in this area could be designed.

The right Common Carotid Artery (CCA) arises from the brachiocephalic trunk immediately posterior to sternoclavicular joint. The left CCA usually arises directly from the aortic arch and has a thoracic and a cervical course. CCA is a large artery that exhibits a stable anatomy in its cervical course without any branches. At its termination it bifurcates to form the larger Internal Carotid artery (ICA) and the relatively smaller External Carotid artery (ECA), generally at the level of $3^{\text {rd }}$ or $4^{\text {th }}$ cervical vertebrae (6-10), which approximately correspond to the upper margin of thyroid cartilage. Carotid bulb is a fusiform dilatation of the proximal ICA just above the bifurcation containing baroreceptors that are concerned with auto regulation of cerebral blood flow and systemic blood pressure. The terms 'high' and 'low' carotid bifurcation are encountered in literature, and has been variably defined. According to some authors high bifurcation is defined as higher than C3/C4 intervertebral discs.(11) 
Variations observed in the CB level has been explained embryologically.(12) Usually, the third foetal aortic arch give rises to CCA, proximal ICA and the ECA with genesis of the remainder of cranial ICA from the foetal dorsal arch. Variations in the origin of the ECA bud within the third arch or directly from dorsal aorta will decides the level of carotid bifurcation.(13,14) If ECA arises proximally, CB position will be low and if it emerges distally vice versa will result. The ICA arising from the second aortic arch and the dorsal aorta with ECA commencing from first arch will lead to a very high position of the carotid bifurcation.

ICA as the larger branch of CCA is the dominant supply to the brain. Its course is divided in to seven segments in the head and neck; namely cervical, petrous, lacerum, cavernous, ophthalmic, clinoid and communicating. Branches do not arise from the cervical course and it is subject to numerous morphological variations such as tortuosity, kinking and coiling.(15) The ICA ultimately terminates as anterior and middle cerebral arteries.

ECA is recognized to show wide variations in length and branching pattern. Length of the common trunk markedly varies and it usually gives rise to the superior thyroid, lingual, facial, ascending pharyngeal, occipital and posterior auricular branches, terminating as superficial temporal and internal maxillary arteries.

In anatomical studies (1-7) CB has been defined in relation to bony points, such as the cervical vertebrae, hyoid bone, thyroid cartilage and angle of mandible. However, among these, the only relatively fixed and hence the most reliable bony point is the cervical vertebrae. Therefore, it is of greater utility in the clinical setting in evaluating the level of carotid bifurcation.

Most available studies have been conducted in cadavers $(7,8,16-18)$, with only a limited number of angiographic studies done in live subjects $(1,9)$ which raises the concern that cadaveric anatomy may be subjective to the post-mortem changes and thus may not represent true dimensions during life. In addition, most available studies were found to have a relatively low number of subjects of around 100 or less.

As stated above, thorough knowledge of various levels of CCA bifurcation and ICA dimensions is critical in head and neck endovascular interventions. However there is very limited data on this anatomic region available from the South Asian subcontinent (6). Similarly data regarding ICA dimensions is sparse, with studies available from Cuba(16), Turkey (18) and New Zealand (17) with no studies available from the region, a gap which this study aims to fulfill.

\section{Methodology}

Convenience sampling was employed to obtain angiographic images of those who presented consecutively for digital subtraction angiography to the Angiography section of Neurotrauma centre, National Hospital of Sri Lanka during the study period from February 2018 to January 2019. Accordingly, 287 patients; 129 males and 158 females, from 6 to 88 years of age were included in our study. Ethics clearance was obtained from the National Hospital of Sri Lanka and approval for the study was obtained from board of study in Radiology, Postgraduate Institute of Medicine, University of Colombo. As per institutional protocols, patient preparation was achieved with standard aseptic technique. Head was oriented in neutral positioning and gentle fixation of the head was achieved with linen-band tightened over the patient's forehead. Inflix ${ }^{\mathrm{TM}}$, bi-planar angiography machine from Toshiba medical imaging was used in image acquisition. 
Conventional common femoral arterial access obtained, preferably from right groin. The departmental practice is to secure the access with $5 \mathrm{~F}$ sheath unless patient is of paediatric age or planning for subsequent intervention. Depending on the patient's age and the orientation of arch vessels, Picard/Vertebral/Simmons catheters of 4-5 F were used to perform sequential extra and intra cranial angiogram with controlled hand injections.

Departmental practice was to perform common carotid runs before proceeding with selective cannulation of internal carotids, which was done to rule out underlying atherosclerotic plaques which demand meticulous canulation technique to prevent thromboembolic complications and for road mapping which enabled swift ICA interrogation. Being a routine step of cerebral angiogram, this projection enables to collect essentially all detail that the study requires without additional radiation or contrast injection (Fig. 1).

The images obtained were installed into Syringo ${ }^{\mathrm{TM}}$ work station and all measurements were taken with auto-calibration mode offered by the service provider. Lateral projection of the common carotid run with bony overlay was used to evaluate the level of carotid bifurcation (CB) in relation to the cervical vertebrae. Thirteen landmarks were identified; C1, C1/2, C2, $\mathrm{C} 2 / 3, \mathrm{C} 3, \mathrm{C} 3 / 4, \mathrm{C} 4, \mathrm{C} 4 / 5, \mathrm{C} 5, \mathrm{C} 5 / 6, \mathrm{C} 6, \mathrm{C} 6 / 7$ and $\mathrm{C} 7$, which correspond to the respective vertebral bodies and intervertebral disc that $\mathrm{CB}$ was projected over. The same lateral CCA projection served to determine the length as well as diameter of mid-cervical ICA and the neck length. (Fig. 1) The length of cervical ICA was measured from bifurcation up to its angulation at the petrous segment. Diameter of the ICA was taken at the mid-point between the bifurcation and the angulation, ensuring that it was measured in a normal segment without atherosclerotic narrowing and it was recorded in millimeters. The vertical distance between the external occipital protuberance and the lower margin of spinous process of C7 vertebra was taken as the neck length and was measured in centimeters (Fig. 2).

All measurement as well as image analysis was done by the same investigator throughout the study to minimize inter-observer variation. Both CCAs were subjected to study unless angiograms were done only for one side. Patients whose head was deviated from the standard neutral position, having a history of regional intervention such as a history of orthopaedic fixation or carotid stenting were excluded from the study. Similarly, subjects with suboptimal and unsaved images were also excluded to minimize measurements errors.

Univariate, bivariate and multivariate analysis of data was performed using IBM SPSS Statistics for Windows version 26.0 statistical package program. Categorical variables were presented as frequency (n) and percent (\%). Data was analysed for normality with Kolomgorov-Smirnov test, which showed non-normally distributed data. Therefore, nonparametric statistical tests were used with Mann-Whitney U test used for comparison of medians of two independent groups and Spearman rho correlation coefficient used for evaluation correlations among variables. Multivariate linear regression analysis was done for independent variables that were found to be significantly correlated with the dependent variable. $\mathrm{P}<0.05$ was regarded as statistically significant.

\section{Results}

The study sample consisted of 287 individuals (in whom a total of 554 CBs were evaluated) with 158 females (55.1\%) ranging from 6 to 88 years (mean - 48.3 years) and 129 males (44.9\%) from 9 to 84 years (mean - 46 years). Only unilateral images were available in 20. The images were analyzed for the level of CB, neck length and cervical ICA dimensions (length and mid cervical diameter) according to the criteria described previously. 
As per the primary objective, bilateral CB levels of the subjects were evaluated with reference to anatomical landmarks in neck. Highest CB level was observed at right C2 level in both males $(n=1,0.4 \%)$ and females $(n=15,4.9 \%)$. The lowest CB in males was seen at left C6/7 level ( $n=1,0.4 \%)$ while in females it was seen slightly higher at C5/6 level $(n=1,0.3 \%)$. CB among males was most frequently seen at C3/4 level $(n=79,31.9 \%)$ while in females it was at C3 level $(n=104,34 \% \%)$ if side isn't considered. Figures 3 and 4 depict the distribution of CBs in male and female subjects.

On the other hand, the commonest level at which CB occurred on the right side in both genders was at C3 level ( $n=102,36.5 \%)$ while on the left it was at C3/4 ( $n=85,30.9 \%)$. C3 was the commonest level $(n=174,31.4 \%)$ at which CB occurred in the study population overall, irrespective of gender or side. When correlated with gender, CB level was found to be significantly lower in males compared to females $(\mathrm{U}=30109.5, \mathrm{p}<0.001)$ and was also lower on left side compared to the right $(U=33850.5, p=0.014)$.

Table 1 shows the distribution of right and left CBs in the 267 study subjects in whom images of both common carotid angiography runs were available. Bilaterally symmetric CBs were seen among 136 subjects (50.9\%) which was commoner among females (M:F of 1:1.3). Symmetric CBs was most frequently observed at C3 level $(n=49,18.4 \%)$. Among the 131 individuals (49.1\%) with asymmetric CBs, a higher right $\mathrm{CB}$ was commoner, with 88 (65.9\%) having a higher right CB while 43 (32.8\%) had a higher left CB. In those with asymmetric CBs in 97 (74.0\%) the difference in levels was only one (eg: CBs at right C3 and left C3/4 or at left $\mathrm{C} 4$ and right C3/4). A difference of two levels (eg: right C3 and left C4) was seen in 30 (22.9\%) and a three-level difference (eg: right C3 and left C4/5) in 11 (8.4\%). A difference in 4 levels, which was the largest difference recorded was seen in a single individual who had the right CB at C3 and left CB at C5.

Table 1: Distribution of carotid bifurcations bilaterally ( $\mathrm{n}=267)$

\begin{tabular}{|c|c|c|c|c|c|c|c|c|}
\hline \multicolumn{2}{|c|}{ Left } & \multirow[b]{2}{*}{ C2/3 } & \multirow[b]{2}{*}{ C3 } & \multirow[b]{2}{*}{ C3/4 } & \multirow{2}{*}{ C4 } & \multirow[b]{2}{*}{ C4/5 } & \multirow[b]{2}{*}{ C5 } & \multirow[b]{2}{*}{ C5/6 } \\
\hline Right & C2 & & & & & & & \\
\hline C2 & $1.5 \%$ & $1.9 \%$ & $0.4 \%$ & - & - & - & - & - \\
\hline C2/3 & $0.7 \%$ & $6.0 \%$ & $4.9 \%$ & $1.1 \%$ & $0.4 \%$ & - & - & - \\
\hline C3 & - & $2.6 \%$ & $18.4 \%$ & $10.5 \%$ & $4.5 \%$ & $0.4 \%$ & $0.4 \%$ & - \\
\hline C3/4 & - & $0.7 \%$ & $2.2 \%$ & $12.4 \%$ & $3.7 \%$ & $1.1 \%$ & - & - \\
\hline C4 & - & - & $1.1 \%$ & $5.2 \%$ & $10.9 \%$ & $2.2 \%$ & $0.7 \%$ & - \\
\hline C4/5 & - & - & - & $0.7 \%$ & $1.5 \%$ & $1.1 \%$ & $0.4 \%$ & - \\
\hline C5 & - & - & - & $0.4 \%$ & $0.7 \%$ & - & $0.7 \%$ & $0.4 \%$ \\
\hline
\end{tabular}

(Symmetric CBs are indicated in bold) 
ICA dimensions were measured due to their importance in endovascular interventions. Average mid-cervical ICA diameter was $4.1 \mathrm{~mm} \pm 0.9 \mathrm{~mm}$ (range $1.0 \mathrm{~mm}-7.6 \mathrm{~mm}$ ) and $4.0 \mathrm{~mm} \pm 0.8 \mathrm{~mm}$ (range of $2.0 \mathrm{~mm}-6.7 \mathrm{~mm}$ ) (Table 2) and ICA length ranged between $3.5 \mathrm{~cm}$ to $12.0 \mathrm{~cm}$ (mean $6.4 \mathrm{~cm} \pm 1.5 \mathrm{~cm}$ ) and $2.9 \mathrm{~mm}$ to $10.9 \mathrm{~mm}$ (mean $5.8 \mathrm{~cm} \pm 1.4 \mathrm{~cm}$ ) in males and female respectively. (Table 3 ). The mean mid-cervical ICA diameter for the total population was $4.0 \mathrm{~mm}$ while the mean ICA length was $6.1 \mathrm{~cm}$.

Table - 2. Distribution of ICA diameter by gender

\begin{tabular}{lllll} 
& Male & & Female & \\
\hline Diameter $(\mathbf{m m})$ & $\mathbf{n}$ & $\mathbf{\%}$ & $\mathbf{n}$ & $\mathbf{\%}$ \\
$\leq 2$ & 1 & 0.6 & 0 & 0 \\
$>2$ to $\leq 3$ & 10 & 6.3 & 15 & 7.5 \\
$>3$ to $\leq 4$ & 71 & 44.7 & 104 & 52 \\
$>4$ to $\leq 5$ & 59 & 37.1 & 56 & 28 \\
$>5$ to $\leq 6$ & 13 & 8.2 & 20 & 10 \\
$>6$ to $\leq 7$ & 3 & 1.9 & 5 & 2.5 \\
$>7$ to $\leq 8$ & 2 & 1.3 & 0 & 0
\end{tabular}

Table - 3. Distribution of ICA length by gender

\begin{tabular}{lllll} 
& Male & & Female & \\
\hline Length $(\mathbf{c m})$ & $\mathbf{n}$ & \% & n & \% \\
$>2$ to $\leq 3$ & 0 & 0 & 1 & 0.2 \\
$>3$ to $\leq 4$ & 7 & 1.2 & 26 & 4.5 \\
$>4$ to $\leq 5$ & 25 & 4.4 & 73 & 12.7 \\
$>5$ to $\leq 6$ & 54 & 9.4 & 103 & 17.9 \\
$>6$ to $\leq 7$ & 72 & 12.5 & 54 & 9.4 \\
$>7$ to $\leq 8$ & 50 & 8.7 & 25 & 4.4 \\
$>8$ to $\leq 9$ & 17 & 3.0 & 10 & 1.7 \\
$>9$ to $\leq 10$ & 11 & 1.9 & 6 & 1.0 \\
$>10$ to $\leq 11$ & 4 & 0.7 & 4 & 0.7 \\
$>11$ to $\leq 12$ & 3 & 0.5 & 0 & 0 \\
\hline
\end{tabular}


The ICA was also found to be significantly longer in males compared to females. ( $U=28560$, $p, 0.001$ ). As anatomically expected, ICA was longer in those with longer necks (Spearman $\rho$ $=0.469, \mathrm{p}<0.001)$ and when the level of CB was lower (Spearman $\rho=0.624, \mathrm{p}<0.001)$. No correlation was found between neck length and the level of CB $(p=0.099)$.

A multiple regression analysis was done to assess if gender, CB level and neck length independently predicted ICA length. The regression model was statistically significant $(\mathrm{F}(3,465)=198.36, \mathrm{p}<0.001)$ and accounted for $55.9 \%$ of the variance of the ICA length $\left(\mathrm{R}^{2}=0.559\right.$ and adjusted $\left.\mathrm{R}^{2}=0.561\right)$. CB level (Beta $\left.=0.522, \mathrm{p}<0.001\right)$ and neck length (Beta $=0.511, \mathrm{p}<0.001$ ) were found to be significant predictors of ICA length and they accounted for approximately $26 \%$ and $25 \%$ of the variance in ICA length respectively. Gender was not found to be a significant predictor of ICA length in the regression model $(p=0.213)$.

\section{Discussion}

Variation of human carotid bifurcation level is a clinically important parameter that has received relatively limited scientific attention. In available literature, the level at which the CB occurs has been described in relation to the various bony reference points. i.e., the hyoid bone, thyroid cartilage, mandible, mastoid, clavicle and cervical vertebrae. However only a few of these are fixed in position with cervical vertebrae being one. Hence it was chosen in our study as the reference point to describe varying positions of CB. A disadvantage of using cervical vertebral levels is that they are not externally visible for pre-operative surface marking. Another difficulty is tracing inter-vertebral levels in patients with cervical spondylosis, especially in the elderly. However cervical vertebrae can be easily demonstrated radiographically with the wide availability of mobile $\mathrm{x}$-ray devices and in-built $\mathrm{C}$ arm devices in surgical theatres, which obviates some of the disadvantages.

CBs have been categorized as high and low, based on the potential for surgical complications and the need for modified surgical approaches with some CB positions. High CBs demands an alternative surgical approach with extensive bony dissection and meticulous dissection techniques in order to prevent accidental injuries to the overriding hypoglossal nerve and cervical ganglion. Similarly, for an interventional radiologist a high CB will narrow the space for catheter manipulation in the resulting shorter cervical ICA due to the increased risk of catheter induced vasospasm if catheter tip is placed too high. In addition, as high CBs and short ECA can coexist (14), extra care is required during ECA embolizations to prevent inadvertent reflux in to ICA. Due to the risk of complications, high bifurcation has been recognized as one of the strongest indications for carotid stenting over endarterectomy as per AHA/ASA guidelines for symptomatic carotid stenosis. (19)

On the other hand, low carotid bifurcation, which have been defined in some studies $(6,10)$ as a bifurcation that lies below the C5 vertebrae, has less clinical significance and is a relatively safe variation. This may have some impact on the anterior surgical approach on cervical discectomies, but is otherwise considered safe for both surgical as well as endovascular interventions.

The level at which CB was found ranged in our study from the highest at C2 (in both males and females) to the lowest at C6/7 in males, while in females it was C5/6. These correspond to a cadaveric study by Kurkcuoglu $A$ et al $(n=100)$ in Turkey (1), which found a similar range of C2 to C6/7, while a CT-angiographic study by McNamara JR et al $(n=86)$ in the Irish population (9) also found a comparable range from lower third of C2 to upper third of C6. However our findings contrasted with the CB level found in the following; study done by Anu VR et al in the Indian population ( $n=95$ ) where CBs ranged from C2 to C5(6), by Klosek SK et al in Thailand ( $\mathrm{n}=43$ ) where it was from C2/3 to C5 (10), and with 2 studies done in 
Japan by Hayashi $\mathrm{N}$ et al $(\mathrm{n}=49)(7)$ and Ito $\mathrm{H}$ et al $(\mathrm{n}=40)(8)$ where it ranged from mid-C2 to $\mathrm{C} 4 / 5$ and from $\mathrm{C} 2$ to $\mathrm{C} 5$ respectively.

C3 was found to be the most frequent level of CB (23.5\%) in the overall study population. However, C3/4 level was the most frequent when only males (31\%) or left sided CBs (30.9\%) were considered while C3 level was more frequent in females (33.3\%) and in right sided CBs (36.5\%). This compares with other studies where mid C3 (7), C3 and C4 $(6,10)$, C4 $(8,9)$ and C4 and C4/5 (1) were found to be commonest levels

Symmetry of CB was found at a rate of $50.9 \%$ in our study with nearly one third of the symmetric CBs found at C3 level. This is within the range of symmetry found in previous studies, which is from $23.4 \%$ (10) to $61.5 \%$ (8), with our findings most closely corresponding to the rate of $52 \%$ from a study done on cadavers in New Zealand (17). We also found that in women CBs are located overall higher and are more likely to be symmetrically located. Additionally, it was found that when CB are asymmetric the higher $\mathrm{CB}$ is more likely on the right side (in both men and women). All of these findings correspond to similar findings by Ito at al. A similar higher prevalence of CB symmetricity in females was also found by Klosek SK et al.(10)

With regard to high and low CBs the levels at which these have been defined is rather variable across literature with high and low CB levels defined as $\geq \mathrm{C} 2$ and $\leq \mathrm{C} 5$ (6), as $\geq$ $\mathrm{C} 2 / 3$ and $\leq \mathrm{C} 4 / 5$ (8), and as $\geq \mathrm{C} 2 / 3$ and $\leq \mathrm{C} 5$ (10). Another common definition of high CBs is those placed higher than C3/4 (11). Alternatively high and low CB have been defined by statistical means by McNamara et al (9) where the highest quartile is defined as a high CB and the lowest quartile as a low $\mathrm{CB}$.

If the above quoted C3/4 level is adopted as the cut-off for high bifurcations, $46.4 \%$ of CBs in our study would be classified as high, which is a relatively large proportion. If the quartile approach is adopted instead, the highest quartile would include CB occurring at C3 and above while lowest would include C4 and below, which would also result in $46.4 \%$ and $28.1 \%$ of all CBs being classified as high and low bifurcations respectively. However, if the relatively higher levels advocated in other studies are used the prevalence of high CBs would decrease to $12.1 \%(\geq C 2 / 3)$ or $2.7 \%$ ( $\geq C 2$ ). Similarly, using a cut-off C4/5 and lower or C5 and lower would reduce low $\mathrm{CB}$ prevalence to $6.9 \%$ and $2.6 \%$ respectively. Few cases of extreme low bifurcation within the superior mediastinum have also been reported but none were encountered in our study. Similarly, none of the subjects were seen to have a CCA that continues as ICA without giving rise to an ECA.

With regards to ICA diameter, its mean in males and females were found to be $4.1 \mathrm{~mm} \pm$ $0.9 \mathrm{~mm}$ and $4.0 \mathrm{~mm} \pm 0.8 \mathrm{~mm}$ respectively, which were somewhat lower compared other studies, where recorded mean diameters were $5.1 \mathrm{~mm} \pm 0.9$ (male) $4.6 \mathrm{~mm} \pm 0.8 \mathrm{~mm}$ (female) in a Polish study (20), $7.3 \mathrm{~mm} \pm 1.3 \mathrm{~mm}$ (male), $6.6 \pm 1.5$ (female) in a Cuban study (16), $6.1 \mathrm{~mm} \pm 1.3 \mathrm{~mm}$ (outer diameter) in a Turkish study (18) and $7.38 \mathrm{~mm}$ in a study done in New Zealand (17). The relatively lower mid ICA diameters found by us may reflect the comparatively smaller physical size of our population. However, a larger study measuring the ICA diameter in two orthogonal projections (producing greater accuracy) may be required to further explore this finding.

Among the variables assessed, mid cervical ICA diameter is the most clinically relevant for stenting. Hence our findings may prove as a useful predictor in local practice in the event of acute occlusions / dissections where the native size cannot be directly determined by angiography, while also facilitating the pre-procedural determination of stent sizes suitable for carotid interventions. 
We were unable to find any correlation between $\mathrm{CB}$ level and neck length which was similar to Kurkcuoglu A et al (1) who reported the same. However, ICA length was found to be positively correlated with the neck length, lower CB levels and male gender. Further statistical evaluation of these factors by multiple regression analysis revealed that only the neck length and CB level were significant independent predictors of ICA length, while gender was not. Given that neck length varies according to gender, we postulate that when both these factors are included in a regression analysis, neck length would better be able to account for the variance in ICA length that would otherwise be accounted for by gender, thus rendering gender a non-significant predictor.

\section{Limitations}

While this is the largest study done so far utilizing DSA, the number of subjects evaluated is however relatively limited. Hence further studies with a larger sample, including paediatric age subjects where possible, will be of benefit in confirming and expanding the ICA dimensions obtained in this study. In addition, this study utilized only images obtained from the lateral plane for measurement of ICA diameter. We are of the view that the accuracy of the values obtained could be further improved if bi-planar images are used and the average of the two planes calculated for each patient. Another limitation of this study was that while the vertical height of cervical ICA were measured, their tortuosity were not assessed, rendering the measurement relatively less accurate as it was only an approximate measurement of ICA length. ICA tortuosity have been measured in several other studies $(9,18,21)$ as it is considered as an anatomic parameter which may have an impact on stenting. Therefor it required further evaluation in the local setting. Furthermore, we have not evaluated the relationship of ECA to ICA and ECA branching variations in this study, and hence we propose that it may be an area of interest for a future study.

\section{Conclusion}

A thorough knowledge of the range of levels at which CB occur will help reduce inadvertent endovascular and surgical complications. Our study, which to our knowledge is the largest DSA study of CB done so far, found that carotid bifurcations were most frequently seen at C3 level, and it was symmetrical in $50.9 \%$ of the study population. with variation according to gender and side of the body, reflecting the available data in the literature in this regard. Range of the level of bifurcation found in our study mirror much of the published data with the highest being at C2 and lowest at C6/7. Cervical ICA mean calibre was $4.0 \mathrm{~mm}$, which was relatively low in our population. ICA diameter was significantly higher in males compared to females among the study subjects. The average length of ICA was $6.1 \mathrm{~cm}$ and this was confounded by tortuosity of the ICA.

\section{Recommendations}

These findings would be helpful when planning and carrying out carotid interventions in local patients. We would like to recommend that a larger study be carried out, which will be able to further investigate the range of ICA calibre in the population. Such a study may also be helpful in evaluating other interesting research questions such as whether height of the patient is related to the level of carotid bifurcation.

\section{Acknowledgments}

Grateful to all of my colleagues and the staff of the angiography section of Neurotrauma Centre, of the National Hospital of Sri Lanka whose steadfast support proved a great strength. All the patients, whose imaging evaluations were utilized in this study, are sincerely acknowledged for their silent contribution. I owe big respect to the guidance given by my teachers as well as the Postgraduate Institute of Medicine, Sri Lanka for the success of this project. 


\section{References}

1. Kurkcuoglu A, Aytekin C, Oktem H, Pelin C. Morphological variation of carotid artery bifurcation level in digital angiography. Folia Morphol. 2015;74(2):206-11.

2. Ranasinghe P, Mathangasinghe Y, Jayawardena R, Hills AP, Misra A. Prevalence and trends of metabolic syndrome among adults in the asia-pacific region: a systematic review. BMC Public Health. 2017;17:101.

3. Coward LJ, Featherstone RL., Brown MM. Safety and Efficacy of Endovascular Treatment of Carotid Artery Stenosis Compared With Carotid Endarterectomy. Stroke. 2005 Apr 1;36(4):905-11.

4. Boulanger M, Camelière L, Felgueiras R, Berger L, Rerkasem K, Rothwell PM, et al. Periprocedural Myocardial Infarction After Carotid Endarterectomy and Stenting. Stroke. 2015 Oct 1;46(10):2843-8.

5. Dangas G, Laird JR, Mehran R, Satler LF, Lansky AJ, Mintz G, et al. Carotid artery stenting in patients with high-risk anatomy for carotid endarterectomy. J Endovasc Ther Off J Int Soc Endovasc Spec. 2001 Feb;8(1):39-43.

6. Anu VR, Pai MM, Rajalakshmi R, Latha VP, Rajanigandha V, D’Costa S. Clinicallyrelevant variations of the carotid arterial system. Singapore Med J. 2007 Jun;48(6):5669.

7. Hayashi N, Hori E, Ohtani Y, Ohtani O, Kuwayama N, Endo S. Surgical anatomy of the cervical carotid artery for carotid endarterectomy. Neurol Med Chir (Tokyo). 2005 Jan;45(1):25-9; discussion 30.

8. Ito H, Mataga I, Kageyama I, Kobayashi K. Clinical anatomy in the neck region--the position of external and internal carotid arteries may be reversed. Okajimas Folia Anat Jpn. 2006 Feb;82(4):157-67.

9. McNamara JR, Fulton GJ, Manning BJ. Three-dimensional computed tomographic reconstruction of the carotid artery: identifying high bifurcation. Eur J Vasc Endovasc Surg Off J Eur Soc Vasc Surg. 2015 Feb;49(2):147-53.

10. Klosek SK, Rungruang T. Topography of carotid bifurcation: considerations for neck examination. Surg Radiol Anat SRA. 2008 Jul;30(5):383-7.

11. Michalinos A, Chatzimarkos M, Arkadopoulos N, Safioleas M, Troupis T. Anatomical Considerations on Surgical Anatomy of the Carotid Bifurcation. Anat Res Int [Internet]. 2016 [cited 2021 Mar 2];2016. Available from: https://www.ncbi.nlm.nih.gov/pmc/articles/PMC4800075/

12. Sadler TW. Langman's medical embryology. 12th ed. United States: Philadelphia: Wolters Kluwer Health/Lippincott Williams \& Wilkins, c2012;

13. Kim CH, Cho YD, Kang H-S, Kim JE, Jung SC, Ahn JH, et al. Anomalous External Carotid Artery-Internal Carotid Artery Anastomosis in Two Patients with Proximal Internal Carotid Arterial Remnants. Korean J Radiol. 2015 Aug;16(4):914-8. 
14. Gluncic V, Petanjek Z, Marusic A, Gluncic I. High bifurcation of common carotid artery, anomalous origin of ascending pharyngeal artery and anomalous branching pattern of external carotid artery. Surg Radiol Anat SRA. 2001;23(2):123-5.

15. Sacco S, Totaro R, Baldassarre M, Carolei A. Morphological variations of the internal carotid artery: Prevalence, characteristics and association with cerebrovascular disease. Int J Angiol Off Publ Int Coll Angiol Inc. 2007;16(2):59-61.

16. Goubergrits L, Affeld K, Fernandez-Britto J, Falcon L. Geometry of the human common carotid artery. A vessel cast study of 86 specimens. Pathol Res Pract. 2002;198(8):54351.

17. Lo A, Oehley M, Bartlett A, Adams D, Blyth P, Al-Ali S. Anatomical variations of the common carotid artery bifurcation. ANZ J Surg. 2006 Nov;76(11):970-2.

18. Ozgur Z, Govsa F, Ozgur T. Anatomic evaluation of the carotid artery bifurcation in cadavers: implications for open and endovascular therapy. Surg Radiol Anat SRA. 2008 Aug;30(6):475-80.

19. Kernan WN, Ovbiagele B, Black HR, Bravata DM, Chimowitz MI, Ezekowitz MD, et al. Guidelines for the prevention of stroke in patients with stroke and transient ischemic attack: a guideline for healthcare professionals from the American Heart Association/American Stroke Association. Stroke. 2014 Jul;45(7):2160-236.

20. Krejza J, Arkuszewski M, Kasner SE., Weigele J, Ustymowicz A, Hurst RW, et al. Carotid Artery Diameter in Men and Women and the Relation to Body and Neck Size. Stroke. 2006 Apr 1;37(4):1103-5.

21. Weibel J, Fields WS. Tortuosity, coiling, and kinking of the internal carotid artery: I. Etiology and radiographic anatomy. Neurology. 1965 Jan 1;15(1):7-7. 


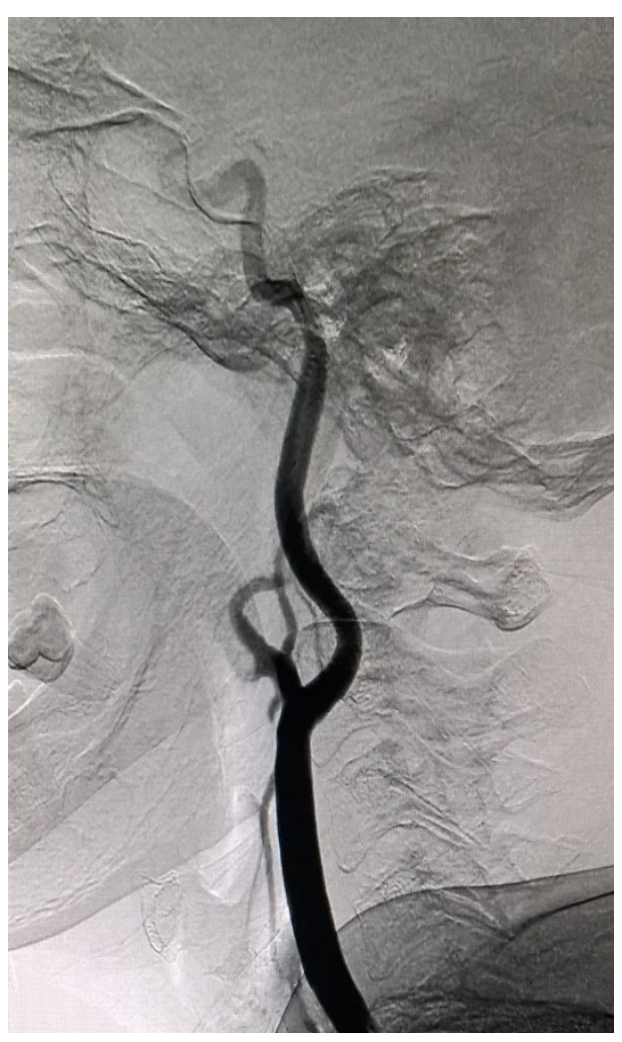

Figure 1. Lateral view of common carotid run with bony overlay

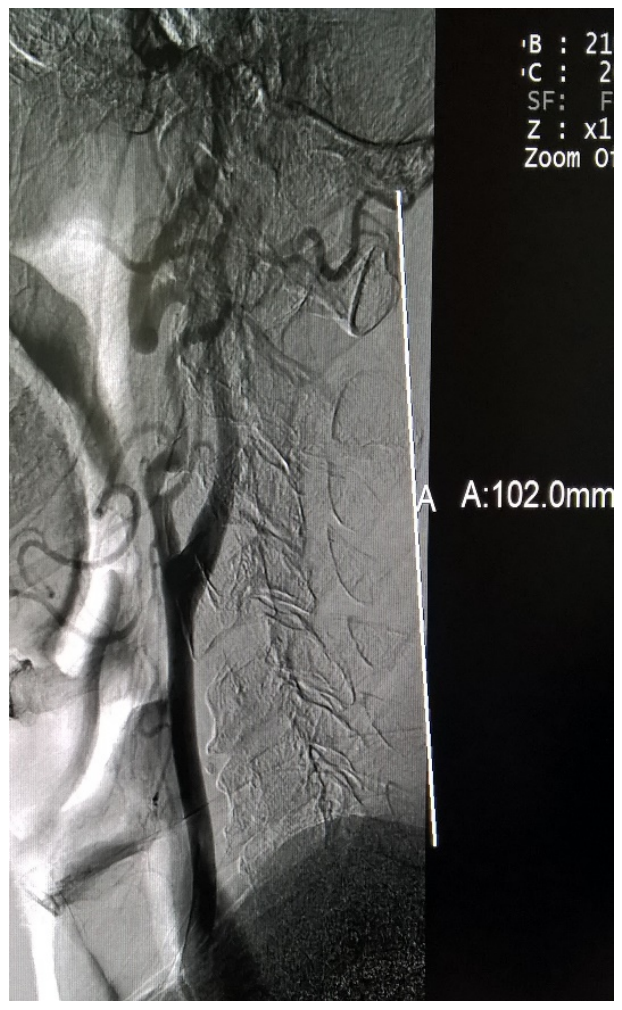

Figure 2. Neck length; from inion to mid C7 spinous process 


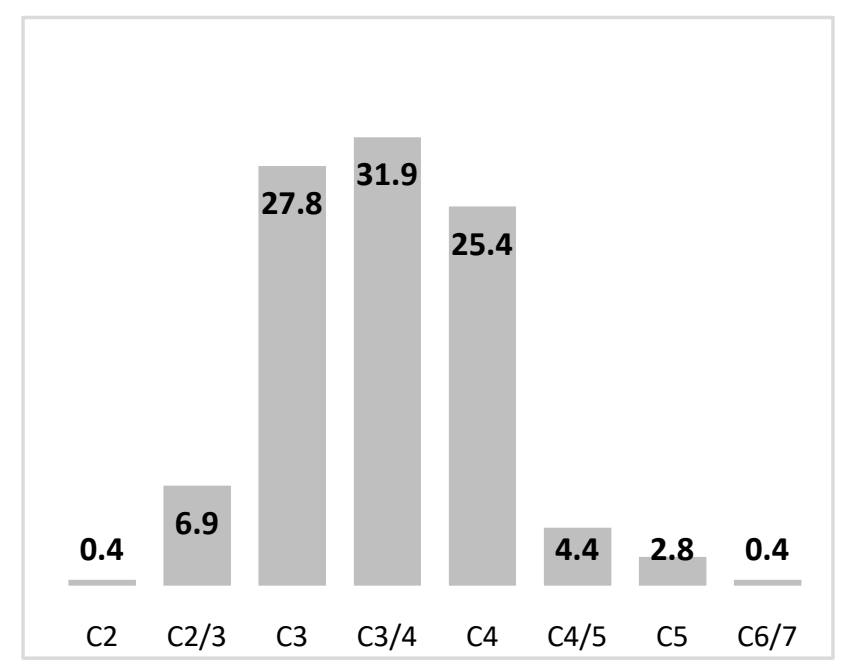

Figure 3a. Percentage distribution Of CB level in males

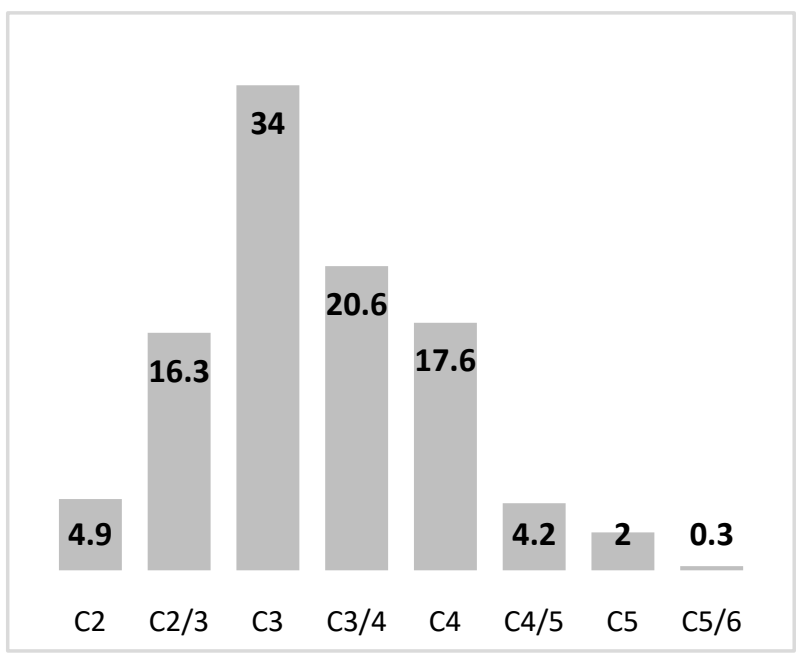

Figure 3b. Percentage distribution Of CB level in females 